\title{
Correction to: Discrimination and Multimorbidity Among Black Americans: Findings from the National Survey of American Life
}

\author{
Hans $\mathrm{Oh}^{1} \cdot$ Joseph Glass ${ }^{2} \cdot$ Zui Narita $^{3} \cdot$ Ai Koyanagi $^{4,5} \cdot$ Shuvam Sinha ${ }^{1} \cdot$ Louis Jacob $^{4,6}$ \\ Published online: 23 June 2020 \\ (C) W. Montague Cobb-NMA Health Institute 2020
}

\section{Correction to: J Racial Ethn Health Disparities} https://doi.org/10.1007/s40615-020-00773-z

The name of author Hans Oh was presented incorrectly as "By Hans Oh" in this article as original published.

The original article has been corrected.

Publisher's Note Springer Nature remains neutral with regard to jurisdictional claims in published maps and institutional affiliations.

The online version of the original article can be found at https://doi.org/ 10.1007/s40615-020-00773-z

Hans Oh

hansoh@usc.edu

1 Suzanne Dworak-Peck School of Social Work, University of Southern California, 1149 Hill St Suite \#1422, Los Angeles, CA 90015, USA

2 Kaiser Permanente Washington Health Research Institute, 1730 Minor Ave, Suite 1600, Seattle, WA 98101, USA

3 Department of Psychiatry, The Johns Hopkins University School of Medicine, Baltimore, MD, USA

4 Research and Development Unit, CIBERSAM, Parc Sanitari Sant Joan de Déu, Barcelona, Spain

5 ICREA, Pg. Lluis Companys, 23, Barcelona, Spain

6 Faculty of Medicine, University of Versailles Saint-Quentin-en-Yvelines, 78180 Montigny-le-Bretonneux, France 